\title{
Numerical study of the stress field during crack growth in porous rocks
}

\author{
Yongming Yang $\cdot$ Yang $\mathrm{Ju} \cdot$ Yeheng Sun • \\ Dongxiao Zhang
}

Received: 20 May 2015/Accepted: 8 September 2015/Published online: 21 September 2015

(C) Springer International Publishing Switzerland 2015

\begin{abstract}
Stress field is one of the primary factors influencing crack growth in rock. Pores significantly affect the distribution features of stress fields in porous rock. Based on the numerical reconstruction of the porous sandstone, we simulate the crack growth in porous sandstone subjected to splitting loads. The influences of the pores on the stress field during crack growth are analyzed. The distribution features of the stress $\sigma_{\mathrm{y}}$ and the evolution of the principal stress $\sigma_{1}$ are disclosed in porous rock. The effects of the stress distribution on the crack growth behavior are discussed. It is shown that during crack growth the maximum value of the principal stress $\sigma_{1}$ gradually increases for all of the porous models. The maximum
\end{abstract}

Y. Yang · D. Zhang

School of Mechanical and Civil Engineering, China University of Mining and Technology, Beijing 100083,

China

Y. Ju

State Key Laboratory for Geomechanics and Deep Underground Engineering, China University of Mining and Technology, Xuzhou 221116, China

Y. Ju ( $\square)$

State Key Laboratory of Coal Resources and Safe Mining, China University of Mining and Technology,

Beijing 100083, China

e-mail: juy@cumtb.edu.cn

Y. Sun

Geological Scientific Research Institute, SINOPEC

Shengli Oilfield Company, Dongying 257015, China values of tensile stress of $\sigma_{\mathrm{y}}$ decrease linearly with porosity increasing. This study presents a way to understand and characterize the distribution features of the stress field and the influences on the crack growth in porous rock.

Keywords Porous structure $\cdot$ Stress field $\cdot$ Crack growth $\cdot$ Sandstone $\cdot$ Numerical analysis

\section{Introduction}

It is important to probe the crack growth mechanism in rock under the action of in situ stress and hydraulic pressure for unconventional oil and gas resource exploitation. Rock is a complex and discontinuous porous medium that contains a large number of pores. Pores significantly affect the crack growth behavior, e.g., the fracturing initial pressure, crack quantity, growth mode, spatial geometry and distribution feature. However, due to the disorderly distribution of pores over a large range of scales in rock, it is difficult to accurately describe the stress field distribution and evolution during crack growth, which makes the intrinsic mechanisms of the crack growth difficult to characterize. A large discrepancy exists between the expected goal and the site application effect on the hydraulic fracture technique.

Due to the influence of pores, the stress distribution and evolution are extremely complex in porous rock 
during crack growth. At present, most research is based on continuum mechanics, i.e., the porous rock is regarded as a type of continuous medium, regardless of pores. On the basis of continuum mechanics, researchers use numerical and experimental methods to reveal the crack growth behavior from the macroscopic point of view. Wong and Einstein observed and characterized crack growth types in Carrara marble under uniaxial compression (Wong and Einstein 2009). Park and Bobet presented a comparison between experimental observations of gypsum specimens under uniaxial compression with open and closed flaws. Three types of cracks were observed (Park and Bobet 2009). Sahouryeh et al. (2002) presented an experimental and analytical investigation into three-dimensional crack growth under biaxial compression of different materials, including transparent resin samples, each with a single embedded disk-like crack. Chen et al. (2003) studied the growth and coalescence of closed joints under uniaxial and biaxial compressive stresses using rock-like material model tests. Zhang et al. studied the crack growth of schistose rock based on a uniaxial compressive test. The stress thresholds under the three conditions of schistose foliation orientation with respect to loading direction (perpendicular, parallel, and $30^{\circ}$ ) were obtained in a progressive failure process (Zhang et al. 2011). Ke et al. (2010) analyzed the crack growth paths in anisotropic rock using boundary elements. Hosseini studied the fractured crack growth behavior on American Barnett shale using linear elastic fracture mechanics and analyzed the impact of crustal stress on the Barnett shale crack growth. The preceding research is valuable and helpful for understanding the crack growth mechanism in rock (Hosseini 2013). Zhao et al. (2013) studied the formation mechanism of the fracture network of shale based on rock dynamics. Quangui et al. (2014) studied the crack growth mechanism subjected to pulse hydraulic fracturing using a three-axis loading experiment. Under a certain load condition, the crack growth is closely related to the stress state in porous rock and pores significantly affect the stress distribution characteristics (Bordia 1971; Chai and Wu 2000; Min and Jing 2004; Pavlov et al. 2010; Giwelli et al. 2013; Feng and Shi 2013).

However, most attentions have been paid to the macroscopic responses of porous rock under external loads. This is a typical "black box" problem, meaning that most researchers focus on the external mechanical responses of rocks with little quantitative interpretation of what happens to the porous structures. The effect of interior pores on the stress field has not been thoroughly studied to interpret the mechanism that governs the complex behavior of porous media. This phenomenological representation ignoring quantitative characterization of porous effects may not be able to accurately describe the stress field distribution and evolution in order to explicate the intrinsic mechanisms affecting crack growth.

Therefore, an accurate and reliable model for porous rock is of critical importance to quantitatively explain how the interior stress field governs the external crack growth of porous rocks. Working with the porous reconstruction model, the relationship between the porous structure and the stress distribution characteristics can be established, and the influence mechanism of the stress field on crack growth can be determined. Recently, researchers have attempted to establish porous rock models by mathematical and numerical methods (Barla 1972; Bryant and Blunt 1992; Lock et al. 2002; Jiao et al. 2003; Okabe and Blunt 2004; Liu et al. 2004; Wang et al. 2005; Ju et al. 2008; Yang et al. 2010; Ju et al. 2013). These models provide an effective way to solve a variety of physical and mechanical "black box" problems related to porous rock.

Natural rock is composed of diverse minerals with a large number of pores. The factors that affect the crack growth are various in porous rocks, e.g., the pore quantity, geometric form, size, connectivity and lithology. Alteration of these factors severely affects the stress state and crack growth behavior. It is difficult to identify the pore effect mechanisms on crack growth inside rock. To resolve the intricate issue, in this paper, we consider one effect factor regardless of other factors, i.e., pore quantity. We focus on the effect of porosity on the stress field associated with crack growth in rock under a split load. Using a CT scanning technique, statistic principles and a self-compiled program, the reconstructed models of porous sandstone are established. We designed various five porous models with different porosities. Among these models, the physical properties of the solid phase, the size and the distribution characteristics of the pores remain consistent. Based on the reconstruction models, the Brazilian split tests are simulated to discuss the stress distribution and evolution 
behaviors during crack growth in sandstone using a numerical method. The primary purpose of this study is to investigate the influence of pores on the crack growth behavior. The other effect factors will be discussed in future research.

\section{Numerical reconstruction models of porous sandstones}

In previous work, the authors studied the geometric and statistic distribution features of pores in sandstone using CT scanning technique and statistic principles (Ju et al. 2008). The previous results showed that the spatial position of pores follows a uniform distribution, the distance of pores satisfies the Gaussian distribution, and the probability density of the pore radius decreases by the exponential law when the pore radius increases. A three-dimensional porous structural model of sandstone was reconstructed using FLAC3D and a self-compiled program. The reconstructed porous model was consistent with natural sandstone in terms of the aforementioned statistic properties of the pores. More details on the 3D reconstruction can be found elsewhere (Ju et al. 2008). According our previous tests, the mean value of porosity of natural sandstone was within 22.9-23.8 \%. To investigate the effects of various numbers of pores, we designed five types of 3D porous models, as shown in Figs. 1 and 2, with a porosity of 0, 3, 7, 15, and $23 \%$. For the numerical models, we adopted the material properties, including strength, elastic modulus, and Poisson's ratio, obtained from the natural sandstone samples with a porosity of $23 \%$ as the properties for the matrices of all the tested porous specimens. It means we did not alter the material properties of solid matrices except the porosity for the sake of comparison. We reconstructed the numerical models with various porosities and identical mechanical properties of solid matrices.

Generally, pores scale from a few millimeters to dozens of micrometers or even nanometers in a rock sample. In is extremely difficult to take account of the effects of all pores with various sizes in simulation. Considering the advantage and benefit of generating discontinuous pores of various sizes and simulating failure of porous rock, we adopted FLAC3D code to determine the stress field distribution and evolution during crack growth. To focus on the effects of pores on the rock performance, considering the minimum size of elements that can be implemented in FLAC3D code, we arbitrarily selected a small volume from a natural sample as a representative volume in the numerical simulation. The sizes of pores and the volume were magnified 10 times simultaneously so that the pore features, i.e., porosity, statistical and distributional parameters, of the numerically reconstructed model were consistent with those of the natural sandstone. More details can refer to the literature (Ju et al. 2008).

On the basis of the reconstructed porous disk models, a series of Brazil split tests were simulated to investigate the influence of the porosity on the stress distribution characteristics during crack growth using the FLAC3D program. The diameter of the 3D disk models is $50 \mathrm{~mm}$ and the height is $25 \mathrm{~mm}$. The shape of the element meshes of the 3D disk model is hexahedron. The elements are endowed with MohrCoulomb material with a shear modulus of $7 \mathrm{GPa}$, volumetric modulus of $26.9 \mathrm{GPa}$, uniaxial tensile

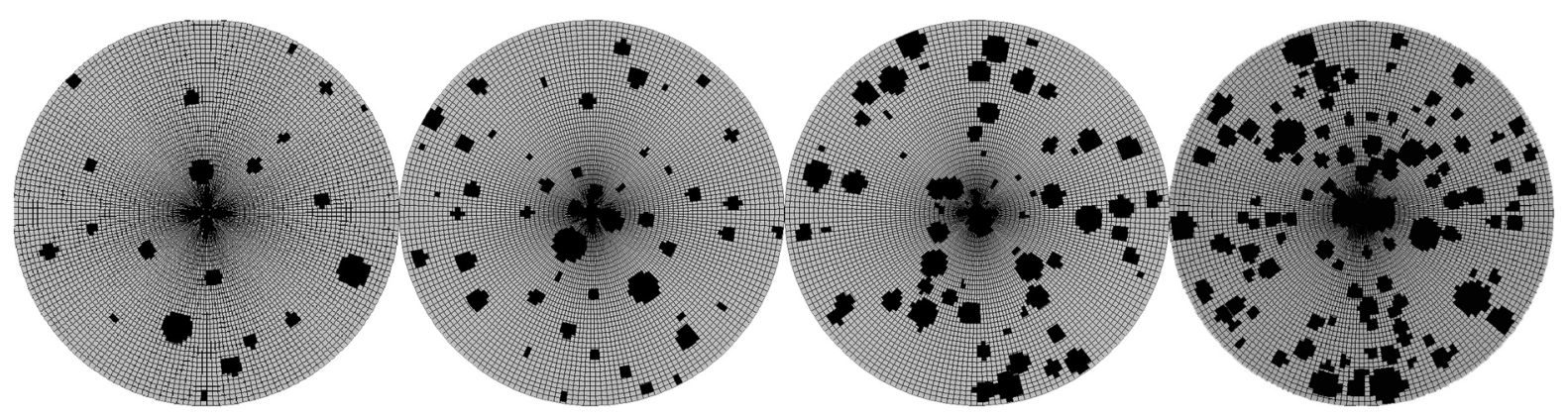

Fig. 1 The 2D reconstructed porous disk model of sandstone. From left to right, the porosities are 3, 7, 15 and $23 \%$. The black spots are the pores 

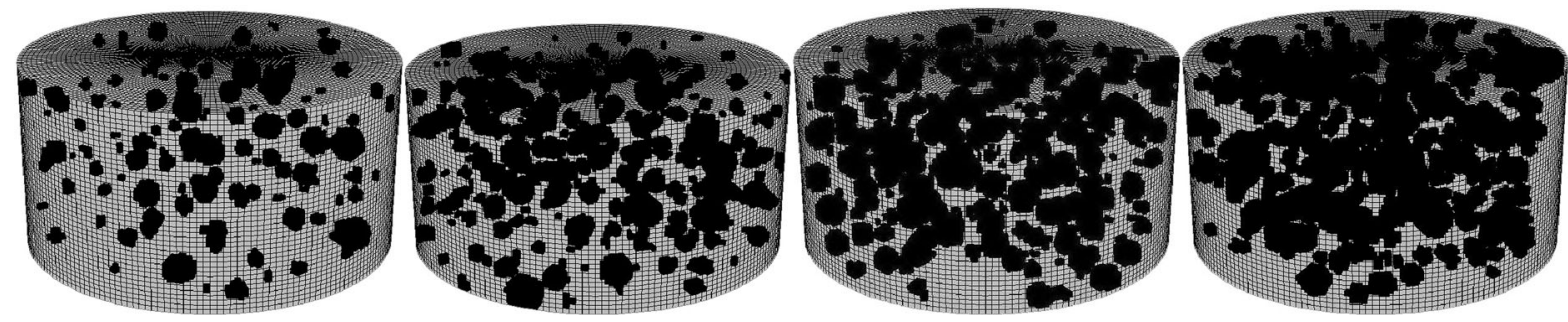

Fig. 2 The 3D reconstructed porous disk model of sandstone. From left to right, the porosities are 3, 7, 15 and $23 \%$. The black spots are the pores

strength of $1.17 \mathrm{MPa}$, cohesion of $27.2 \mathrm{MPa}$ and friction angle of $30^{\circ}$. As aforementioned, the material parameters in the simulation are determined based on the preliminary tests of the natural porous sandstone with a porosity of $23 \%$. We reconstructed the numerical models with different porosities but identical material properties of solid matrices. The line loads are imposed on both the right side and left side of the disk models, along the $\mathrm{X}$-axis direction. The other boundaries are free of constraint. To emphasize the porosity effect, the material parameters and boundary conditions are consistent among all disk models. Figure 3 illustrates the loading pattern. To simulate crack growth, we took a Mohr-Coulomb failure criterion to determine if and where the elements reached failure state in FLAC3D studies. We regarded all the areas that the failed elements occupied and gradually connected as crack growth. We adopted different color to distinguish the failed elements representing cracks from the other elements representing solid matrices.

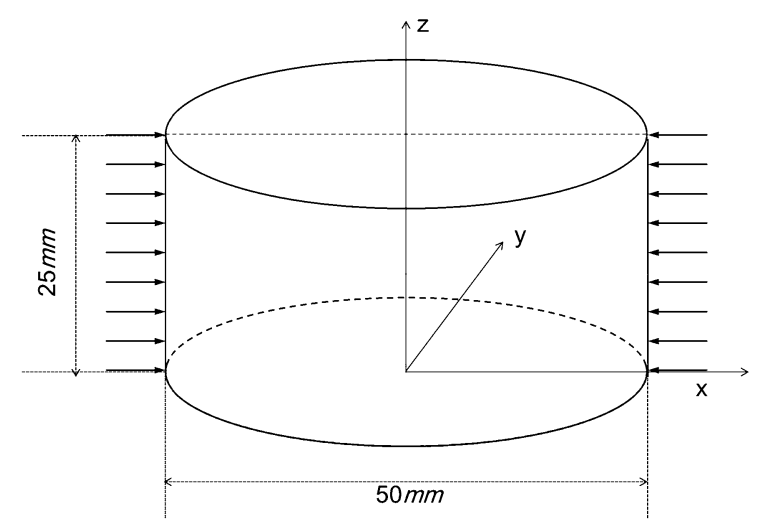

Fig. 3 Illustration of loading patterns

\section{Stress distribution characteristics during crack growth in the porous models}

\subsection{The principal stresses}

To study the stress distribution and evolution during crack growth, we present the stepwise results at different loading steps of peak load in the following figures. It is shown that when load reaches the peak value, all the areas that the failed elements occupied were gradually connected. We referred the progressive connection of failed elements as the process of "crack growth". For the sake of analyzing the crack growth, we chose three representative stages of the crack growth process, which were referred to as crack initiation, crack development, and crack coalescence, respectively. Figure 4 illustrates the distribution of the normal stress $\sigma_{\mathrm{y}}$ on the cross-sections of the disk models at three representative stages. The crosssections are in the middle position along height direction of the disk models. The tensile stress is positive (red regions) and the compressive stress is negative (other color regions). Figure 5 show the normal stress $\sigma_{\mathrm{y}}$ distributing on the longitudinal profiles along loading direction.

The numerical results indicate the follows:

(1) At crack initiation stage, for 3D disk models without pores, i.e., the porosity is $0 \%$, the compressive stress of $\sigma_{y}$ occupies most of the regions of the disk model, and the tensile stress lies in small regions near the loading lines. In the case of $3 \%$ porosity, the $\sigma_{\mathrm{y}}$ distribution features have significant differences compared to the disk model with no pores. The compressive stress of $\sigma_{\mathrm{y}}$ still dominates most of the regions, but the tensile stress appears not only in the small regions nearby loading lines, but also in the 


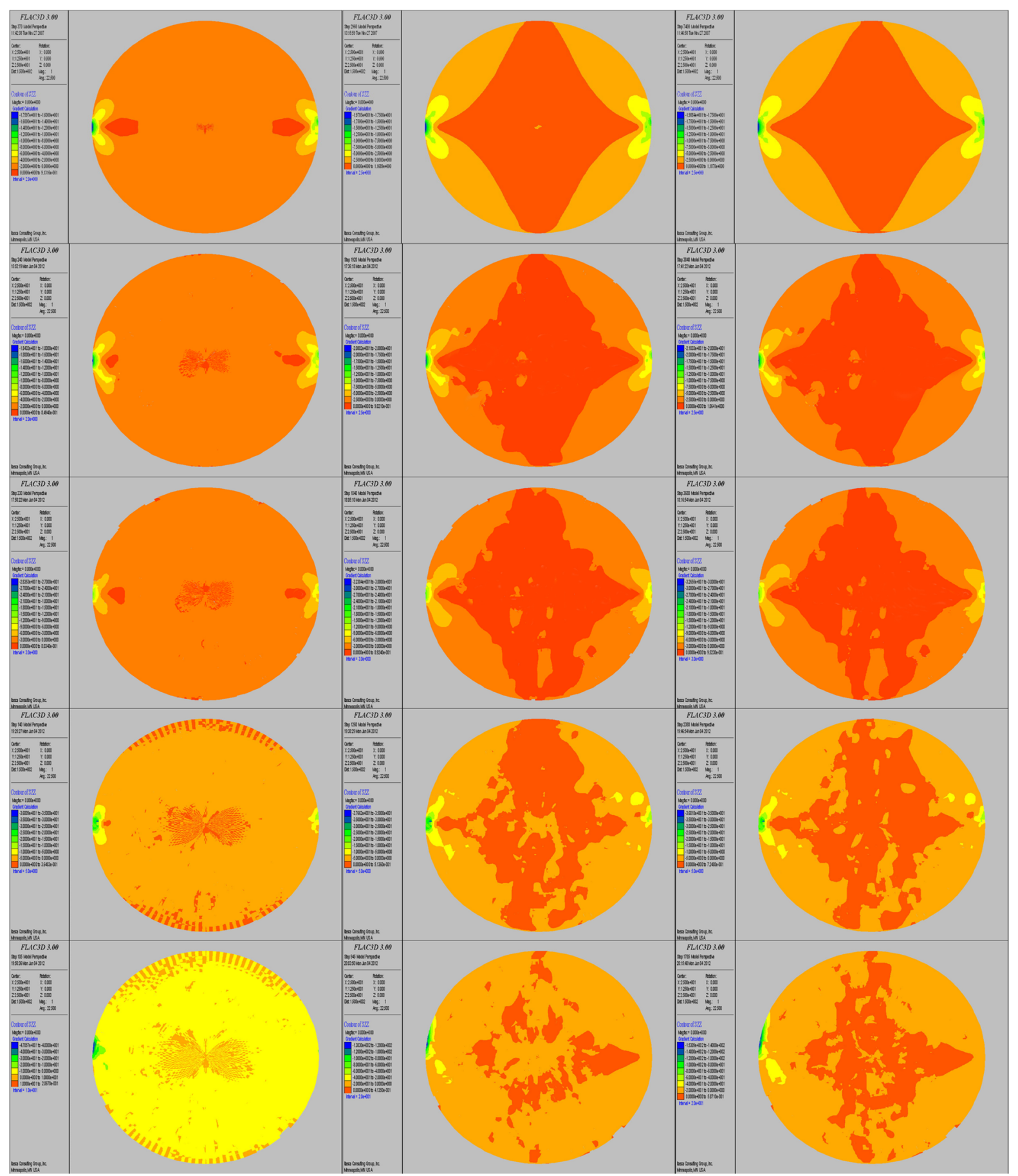

Fig. 4 The distribution of normal stress $\sigma_{y}$ on the crosssections of the porous disks during crack growth. The rows from top to bottom present the results with porosities of $0,3,7,15$ and center regions of the disk model. With porosity increasing, the tensile stress regions gradually enlarge. When the porosity reaches $15 \%$ or
$23 \%$. The columns from left to right present the results at three crack growth stages, i.e., crack initiation, crack development, and crack coalescence

more, the tensile stress is located in the circumference and the center regions of the disk models instead of in the regions nearby loading lines. 


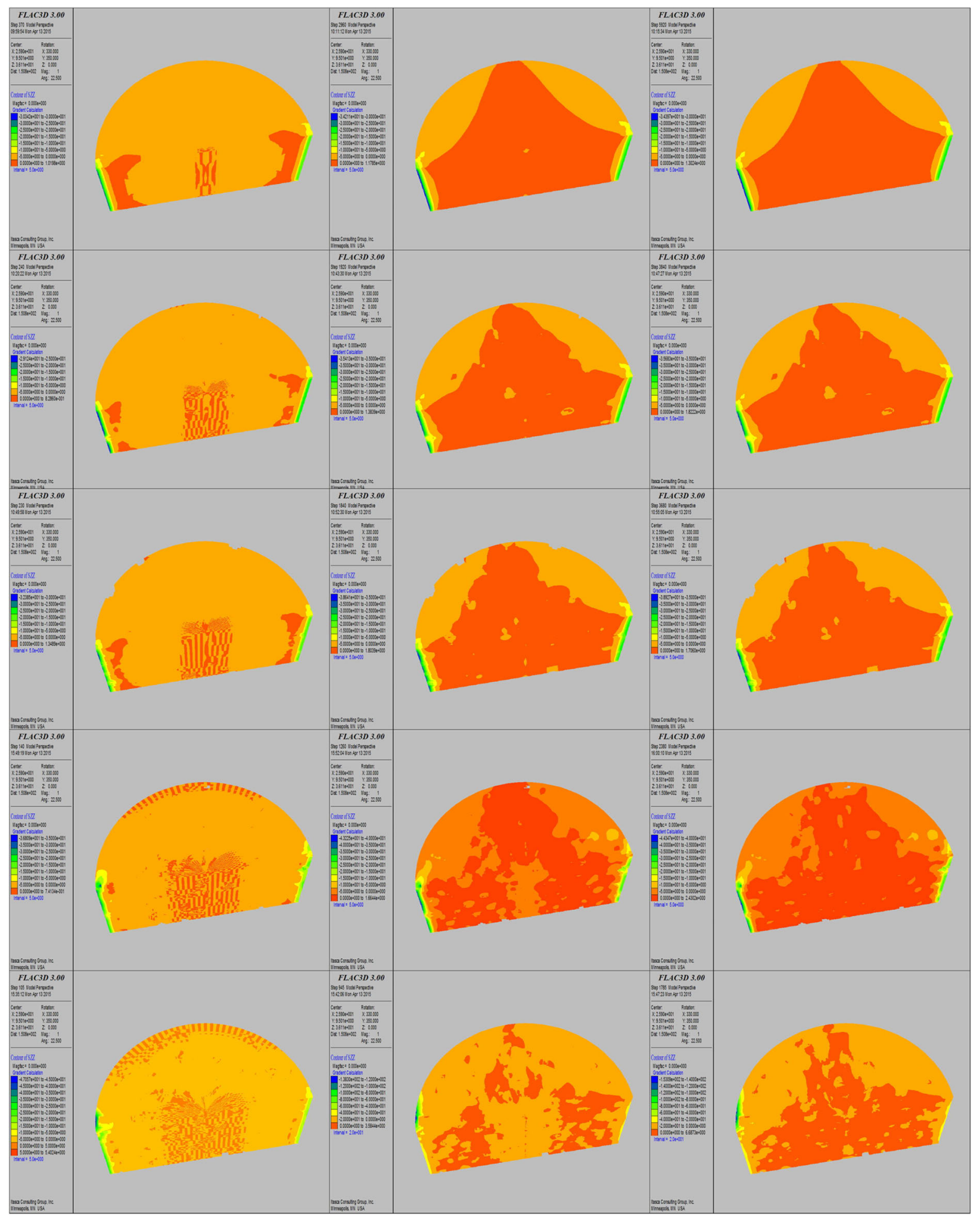

Fig. 5 The distribution of normal stress $\sigma_{\mathrm{y}}$ on the longitudinal profiles of the porous disks during crack growth. The rows from top to bottom present the results with porosities of $0,3,7,15$ and
$23 \%$. The columns from left to right present the results at three crack growth stages, i.e., crack initiation, crack development, and crack coalescence 
(2) At crack development stage, the distribution of the tensile and the compressive stresses present considerable difference as compared to those at the previous stage. For the model free of pores, the tensile stress of $\sigma_{\mathrm{y}}$, rather than compressive stress, dominates most parts of the disk models. The tensile stress regions distribute symmetrically about the diameter along $\mathrm{X}$-axis direction in cross-sections. The compressive stress regions appear only near the loading lines and the four corners of the disk models. With regard to the porous disk models, in contrast to the disk model with no pores, the symmetry of the tensile stress regions disappears. The regions of tensile stress are smaller and concentrated near the loading lines. The compressive stress regions occupy most regions. The regions of tensile stress are smaller for disk models with higher porosity.

(3) At crack coalescence stage, compared to crack growth stage, for the disk models free of pores, the distribution characteristics of the stress change little. The bud-shaped tensile stress regions still play a leading role and distribute symmetrically about the diameter along $\mathrm{X}$-axis. The compressive stress regions appear near the loading lines and the four corners of the disk model. For porous models, the tensile stress regions begin to dominate most of regions and present symmetry about the diameter along $\mathrm{X}$-axis. Because of the effect of pores, the symmetry of tensile stress regions weakens. The tensile stress regions still resemble buds. The distribution features of compressive stress have a little change. With increasing of the porosity, the regions of tensile stress gradually diminish and the symmetry gradually disappears. Simultaneously, there are some small compressive stress regions appearing in the tensile stress regions. The tensile stress regions and compressive stress regions present an interlaced distribution. The higher the porosity is, the more obvious the interlaced phenomenon is. For the disk models with $23 \%$ porosity, the tensile stress regions have no symmetry and bud shape. The compressive stress occupies mostly the same regions compared with the tensile stress.

In summary, the pores significantly affect the stress distribution in porous sandstone during crack growth.
There are large discrepancies among disk models with different porosities in terms of the stress $\sigma_{\mathrm{y}}$ distribution features. At the crack initiation stage, the compressive stress of $\sigma_{\mathrm{y}}$ occupies most regions, and the tensile stress lies only in small regions in all porous disk models. Due to the effect of pores, the distribution range of the tensile stress of $\sigma_{y}$ in the higher porosity models is larger than in lower porosity models. At crack growth, the stress distribution features have larger change. For the disk models with no pores or lower porosities, the tensile stress $\sigma_{\mathrm{y}}$ begins to dominate most regions of the disk models. The tensile stress regions with a bud shape distribute are symmetrical about the diameter along $\mathrm{X}$-axis in the crosssection. The compressive stress regions appear only near the loading lines and the four corners. However, for the disk models with higher porosity, the compressive stress occupies most regions. The tensile stress regions are smaller and have no symmetry. At crack coalescence stage, for the disk models with no pores or lower porosity, the distribution features of stress $\sigma_{\mathrm{y}}$ are consistent with ones at crack growth stage. The tensile stress distributes symmetrically in most of regions and presents a bud shape. The compressive stress occupies a few regions. For higher porosity disk models, the tensile stress regions begin to enlarge and occupy approximately half of the disk models. Due to the effect of pores, the tensile stress regions and the compressive stress regions appear crosswise in the disk models.

It should be noted that the failed areas that we observed in the simulation, which are referred to as the crack growth areas, actually comprise two types of failure elements, i.e., compression-shear-induced failure elements and tension-induced failure elements. The tension-induced failure elements initiated from the neighborhood near the center of the sample, while the compression-shear-induced failure elements took place near the loading ends of the sample.

\subsection{The maximum tensile stress}

For the no pores disk model, the maximum tensile stress of $\sigma_{\mathrm{y}}$ is $1.17 \mathrm{MPa}$. The stress appears on the axis of symmetry and is constant along the axis of symmetry. For porous disk models, because of the effect of pores, the maximum tensile stress of $\sigma_{y}$ has great change. With increasing of porosity, the 
maximum value gradually deviates from the symmetry axis and exist in the pore-intensive region. The higher porosity induces a larger deflected range. The maximum values of tensile stress gradually decrease with increase of porosity. For the model with the porosity of $3 \%$, the maximum value is $1.059 \mathrm{MPa}$. It is $0.936 \mathrm{MPa}$ for $7 \%$, it is $0.707 \mathrm{MPa}$ for $15 \%$, and it is $0.552 \mathrm{MPa}$ for $23 \%$. Compared with the no pores disk model, the tensile stress maximum values of porous models decline by $9.49,19.7,39.5$ and $52.85 \%$. The decline percent gradually increases following a linear relationship with increase of porosity. Figure 6 shows the change curve of the maximum tensile stress with increasing porosity.

Through the above analysis, we can conclude that the failure mode is tension-induced failure for the disk models with no pores or lower porosity. A primary fractured crack passes through the disk model along the diameter of the $\mathrm{X}$-axis direction. When the porosity is higher, the failure mode changes to tension-shear-induced failure. Multiple fractured cracks pass through the disk models instead of a single primary crack.

\section{Evolution of the principal stress $\sigma_{1}$ during crack growth}

To analyze the evolution of the principal stress $\sigma_{1}$ during crack growth, we choose five representative connection processes of failed elements as different "crack growth stages", i.e., crack initiation, growth

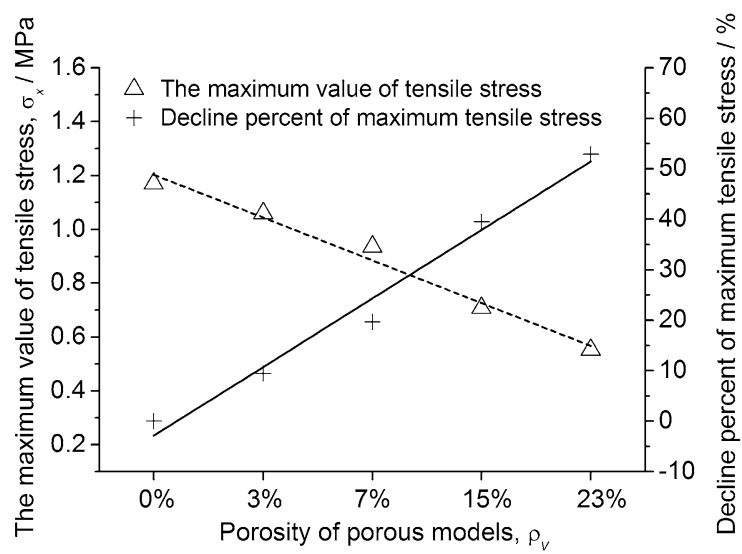

Fig. 6 Relationship curves between the maximum tensile stress of $\sigma_{\mathrm{y}}$ and porosity stage 1, growth stage 2 , growth stage 3 , and crack coalescence. Figure 7 plots the $\sigma_{1}$ distribution in the cross-sections at five crack growth stages. The crosssection is in the middle position along height direction of the porous disk models. Figure 8 gives the evolution curves of the maximum values of $\sigma_{1}$ during crack growth.

During crack growth, the maximum values of the principal stress $\sigma_{1}$ gradually increase. The models with various porosities have different amplifications. The maximum values of $\sigma_{1}$ increase by $13,14.5,16.8$, 17 and $17.5 \%$, respectively, corresponding to the disk models with porosities of $0,3,7,15$ and $23 \%$. The larger the porosity is, the larger the amplification is. At crack coalescence stage, for the disk model free of pores, the maximum value of $\sigma_{1}$ is $1.866 \mathrm{MPa}$. It is $2.176 \mathrm{MPa}$ for $3 \%$ porosity, $2.402 \mathrm{MPa}$ for $7 \%$ porosity, $2.901 \mathrm{MPa}$ for $15 \%$ porosity and $3.246 \mathrm{MPa}$ for $23 \%$ porosity. Pores cause serious stress concentration in the porous models, which leads to that the higher porosity disk models have larger value and more irregular distribution feature of the principal stress $\sigma_{1}$ in contrast to the disk models with smaller porosity. For higher porosity models, the serious stress concentration phenomenon induces multiple fractured cracks appearing in the porous models. In contrast, for lower porosity models or no pores model, the stress concentration phenomena are inconspicuous. It results in only one primary fractured crack passing through the disk model.

\section{Conclusions}

Based on the numerical reconstruction of porous sandstone, we performed a numerical simulation of stress distribution and evolution during crack growth in the rock specimens. The influences of pores on the stress distribution and evolution are probed. It is shown that the influences of pores on the stress distribution and evolution during crack growth have been well characterized and visualized by using numerical reconstruction models and FLAC3D simulation.

(1) The pores significantly affect the stress distribution in porous sandstone during crack growth. There are large discrepancies among disk models with different porosities in terms of 


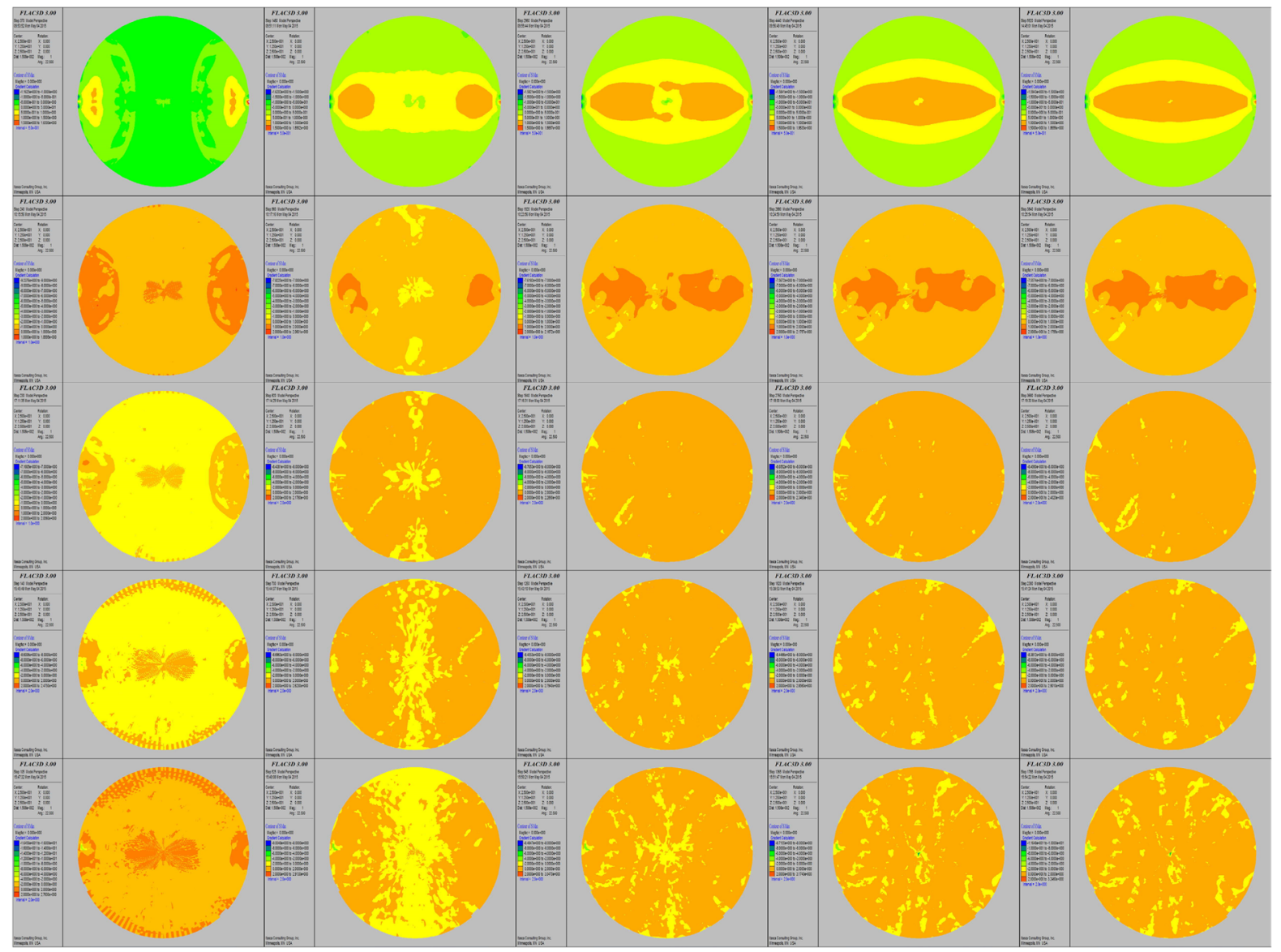

Fig. 7 The principal stress $\sigma_{1}$ distribution on the cross-sections of the $3 \mathrm{D}$ porous disks during crack growth. The rows from top to bottom provide the results with porosities of $0,3,7,15$, and

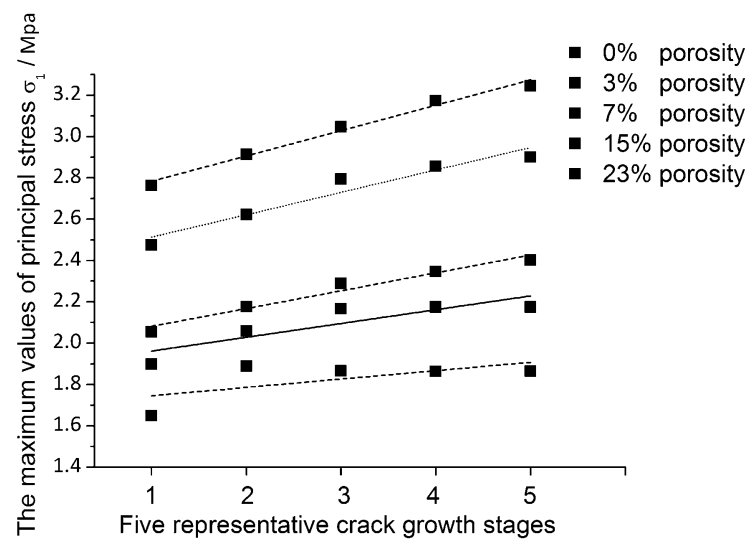

Fig. 8 The evolution curves of the maximum values of $\sigma_{1}$ in cross-section during crack growth
$23 \%$. The columns from left to right present the results at five crack growth stages the distribution features of the tensile stress and compressive stress. In the process of crack growth, the tensile stress of $\sigma_{\mathrm{y}}$ occupies most regions and the compressive stress $\sigma_{y}$ exists in a few regions in the lower porosities models. The tensile stress $\sigma_{\mathrm{y}}$ distributes symmetrically in disk models and presents a bud shape. However, due to the effect of pores, for higher porosity models, the tensile stress occupies approximately half of the disk models until crack coalescence. The tensile stress and compressive stress regions exist crosswise in the disk models. The symmetry of the tensile stress completely disappears.

(2) At crack coalescence stage, for the no pores disk models, the maximum tensile stress $\sigma_{\mathrm{y}}$ appears 
on the axis of symmetry and is constant along the axis of symmetry. For the porous disk models, the maximum tensile stress $\sigma_{\mathrm{y}}$ differs greatly. With increase of porosity, the maximum values of the tensile stress gradually deviate from the symmetry axis and exist in the pore-intensive region. The bigger the porosity is, the larger the deflected range is. The maximum values decrease according to a linear function with increasing porosity.

(3) During crack growth, the maximum values of the principal stress $\sigma_{1}$ gradually increase for all porous disk models. The disk models with various porosities have different amplifications. The larger the porosity is, the larger the amplification is. Pores cause significant stress concentration in the porous disk models, which leads to the disk models with higher porosities having larger values and more irregular distribution features of the principal stress $\sigma_{1}$ compared to the disk models with smaller porosities.

(4) The failure mode is tension-induced failure for the disk models with no pores or lower porosity. A primary fractured crack passes through the disk model along the diameter of $\mathrm{X}$-axis direction. When the porosity is higher, the failure mode changes to tension-shear-induced failure and multiple fractured cracks pass through the disk models instead of a single primary crack.

\begin{abstract}
Acknowledgments The authors are grateful to the National Natural Science Funds for Distinguished Young Scholars of China (Grant No 51125017), the National Natural Science Foundation of China (Grant No 51374213), the 2012 Innovative Talents Program of Jiangsu Province, the Science Fund for Creative Research Groups of the National Natural Science Foundation of China (Grant No 51421003), the Priority Academic Program Development of Jiangsu Higher Education Institutions (PAPD), and the Open Research Project of State Key Laboratory of Coal Resources and Safe Mining (Grant No SKLCRSM13KFB09) for their financial supports.
\end{abstract}

\section{References}

Barla G (1972) The distribution of stress around a single underground opening in a layered medium under gravity loading. Int $\mathrm{J}$ Rock Mech Min Sci Geomech Abstr 9(1):127-154
Bordia SK (1971) The effects of size and stress concentration on the dilatancy and fracture of rock. Int J Rock Mech Min Sci Geomech Abstr 8(6):629-669

Bryant S, Blunt M (1992) Prediction of relative permeability in simple porous media. Phys Rev A 46:2004-2011

Chai JR, Wu YQ (2000) Research on multiple-level fracture network model for coupled seepage and stress fields in rock mass. Chin J Rock Mech Eng 19(6):712-717

Chen WZ, Li SC, Zhu WS, Qiu XB (2003) Experimental and numerical research on crack growth in rock under compression. Chin J Rock Mech Eng 22(1):18-23

Feng YJ, Shi XW (2013) Hydraulic fracturing process: roles of in situ stress and rock strength. Adv Mater Res 616-618: $435-440$

Giwelli AA, Matsuki K, Sakaguchi K, Kizaki A (2013) Effects of non-uniform traction and specimen height in the direct shear test on stress and deformation in a rock fracture. Int $\mathrm{J}$ Numer Anal Methods Geomech 37(14):2186-2204

Hosseini SM (2013) On the linear elastic fracture mechanics application in Barnett shale hydraulic fracturing. In: 47th US rock mechanics/geomechanics symposium, vol 3, pp 2087-2094

Jiao CH, Wang XS, Cai JH, Geng B (2003) Double pores system's effect on acoustic transit time measurements and porosity quantification methods in carbonate formation. Well Logging Technol 27(4):288-290

Ju Y, Yang YM, Song ZD, Xu WJ (2008) A statistical model for porous structure of rocks. Sci China Ser E Technol Sci 51(11):2040-2058

Ju Y, Yang YM, Peng RD, Mao LT (2013) Effects of pore structures on static mechanical properties of sandstone. J Geotech Geoenviron Eng 139(10):1745-1755

Ke JZ, Xu SM, Chen ZX, Ji SY (2010) Boundary element analysis of crack growth paths in anisotropic rock. Chin J Rock Mech Eng 29(1):34-42

Liu TY, Xiao LZ, Fu RS, Wang ZD (2004) Applications and characterization of NMR relation derived from spherecapillary model. Chin J Geophys 47(4):663-671

Lock PA, Jing XD, Zimmerman RW, Schlueter EM (2002) Predicting the permeability of sandstone from image analysis of pore structure. J Appl Phys 92(10):6311-6319

Min KB, Jing L (2004) Stress dependent mechanical properties and bounds of Poisson's ratio for fractured rock masses investigated by a DFN-DEM technique. Int J Rock Mech Min Sci 41(suppl.1):131-137

Okabe H, Blunt MJ (2004) Prediction of permeability for porous media reconstructed using multiple-point statistics. Phys Rev E 70(6):66135-66145

Park CH, Bobet A (2009) Crack coalescence in specimens with open and closed flaws: a comparison. Int J Rock Mech Min Sci 46(2):819-829

Pavlov VA, Martynuk PA, Serdyukov SV (2010) The hydraulic fracture opening pressure multiple test for the stress state measurement in permeable rock. In: Rock stress and earthquakes-proceedings of the 5th international symposium on in situ rock stress, pp 167-172

Quangui L, Baiquan L, Cheng Z (2014) The effect of pulse frequency on the fracture extension during hydraulic fracturing. J Nat Gas Sci Eng 21(11):296-303 
Sahouryeh E, Dyskin AV, Germanovich LN (2002) Crack growth under biaxial compression. Eng Fract Mech 69(18):21872198

Wang BS, Chen Y, Ge HK, Song LL, Wong TF (2005) A discrete element model of porous rock deformation. Chin $\mathrm{J}$ Geophy 48(6):1336-1342

Wong LNY, Einstein HH (2009) Systematic evaluation of cracking behaviour in specimens containing single flaws under uniaxial compression. Int J Rock Mech Min Sci 46(2):239-249
Yang YM, Ju Y, Wang HJ (2010) Physical model and failure analysis of porous rock. Chi J Geotech Eng 32(5):736-744

Zhang XP, Wang SJ, Han GY, Zhang B (2011) Crack growth study of rock based on uniaxial compressive test-a case study of schistose rock. Chin J Rock Mech Eng 30(9):1772-1781

Zhao HF, Chen H, Liu GH, Li YW, Shi J, Ren P (2013) New insight into mechanisms of fracture network generation in shale gas reservoir. J Pet Sci Eng 110(10):193-198 